Abstract 018 Table 1

\begin{tabular}{lcclll}
\hline Variable & $\begin{array}{l}\text { Mean (SD) } \\
\text { Baseline }\end{array}$ & $\begin{array}{l}\text { Mean (SD) } \\
\text { Day 3 }\end{array}$ & $\begin{array}{l}\text { Mean (SD) } \\
\text { Follow-up }\end{array}$ & $\begin{array}{l}\text { Change (95\% CI), } \\
\text { Day 3-Baseline }\end{array}$ & $\begin{array}{l}\text { Change (95\% CI), p } \\
\text { Follow-up-Baseline }\end{array}$ \\
\hline LVEF \% & $72.7(4.7)$ & $71.6(4.2)$ & $70.1(5.2)$ & $-1.1(-2.440$ to 0.16$) \mathrm{p}=0.09$ & $-2.6(-3.97$ to -1.36$) \mathrm{p}=0.0002$ \\
LV Mass g/m & $105.6(12.3)$ & $109.1(12.9)$ & $104.2(13.8)$ & $3.5(1.98$ to 4.55$), \mathrm{p}=0.00004$ & $-1.4(-3.03$ to -1.02$), p=0.31$ \\
RVEF \% & $67.9(5.4)$ & $67.5(5.8)$ & $66.5(5.3)$ & $-0.4(-1.71$ to 0.85$) \mathrm{p}=0.5$ & $-1.4(-2.41$ to -0.43$) \mathrm{p}=0.006$ \\
ERGE & $2.36(0.8)$ & $2.74(1.1)$ & NA & $0.37(0.13$ to 0.62$) \mathrm{p}=0.003$ & $\mathrm{NA}$ \\
STIR & $63.3(19.8)$ & $67.1(20.0)$ & $\mathrm{NA}$ & $3.8(-0.35$ to 8.02$) \mathrm{p}=0.07$ & $\mathrm{NA}$ \\
\hline
\end{tabular}

cancer, Herceptin use increases this risk. Susceptibility is highly idiosyncratic. Although detection of cardiomyocyte injury using endomyocardial biopsy is the gold standard, it is not appropriate for routine monitoring. Serial measurements of LV ejection fraction (LVEF) only detect cardiotoxicity after significant damage has been incurred. Cardiovascular magnetic resonance (CMR) can detect myocardial inflammation and oedema using STIR (Short TI Inversion Recovery) and early gadolinium relative enhancement (EGRE). We hypothesised these CMR sequences could be used as non-invasive tests to assess acute injury and predict cAC.

Methods Patients receiving adjuvant chemotherapy for early breast cancer, were scanned before and 3 days after their first cycle of epirubicin. Follow-up CMR was performed $>1$ year after the final dose of anthracycline, or $>3$ months after the end of Herceptin. Cine imaging was used to measure LVEF; STIR and EGRE images were obtained to assess cardiac inflammation.

Results 51 patients completed the protocol. Changes in CMR parameters are outlined in the Abstract 018 table 1 below. In patients with a $i\{5 \%$ decrease in LVEF $(n=22)$ at follow-up, the day 3 mean EGRE increased by $32 \%(p=0.002)$ and mean STIR SI increased by $15 \%(p=0.003)$. In the remaining patients $(n=29)$ there were no significant changes in EGRE or STIR.

Conclusions/Implications This study has shown that subclinical myocarditis occurring after the first exposure to anthracycline can be detected using CMR and is associated with late falls in LVEF. Thus potentially identifying those at increased risk of premature heart failure, before the majority of damage is caused. Recognition of this susceptibility could inform treatment decisions and/or identify those requiring greater cardiac surveillance.

\section{DEVELOPMENT AND VALIDATION OF A NOVEL PRESSURE- ONLY INTRA-CORONARY INDEX OF CORONARY STENOSIS SEVERITY}

doi:10.1136/heartjnl-2012-301877b.19

${ }^{1} \mathrm{~S}$ Sen, ${ }^{* 2} \mathrm{~J}$ Escaned, ${ }^{1} \mathrm{I}$ Malik, ${ }^{1} \mathrm{G}$ Mikhail, ${ }^{1} \mathrm{R}$ Foale, ${ }^{2} \mathrm{R}$ Mila, ${ }^{1} \mathrm{~J}$ Tarkin, ${ }^{1} \mathrm{R}$ Petraco, ${ }^{1} \mathrm{C}$ Broyd, ${ }^{1} \mathrm{R}$ Jabbour, ${ }^{1} \mathrm{~A}$ Sethi, ${ }^{1} \mathrm{C}$ Baker, ${ }^{1} \mathrm{M}$ Bellamy, ${ }^{1} \mathrm{M}$ Al-Bustami, ${ }^{1} \mathrm{D}$ Hackett, ${ }^{1} \mathrm{M}$ Khan, ${ }^{1} \mathrm{D}$ Lefroy, ${ }^{1} \mathrm{~K}$ Parker, ${ }^{1} \mathrm{~A}$ Hughes, ${ }^{1} \mathrm{D}$ Francis, ${ }^{1} \mathrm{C}$ Di Mario, ${ }^{1} \mathrm{~J}$ Mayet, ${ }^{1} \mathrm{~J}$ Davies. ${ }^{1}$ Imperial College London, London, UK; ${ }^{2}$ Cardiovascular Institute, Hospital Clínico San Carlos, Spain

Background Assessment of stenosis severity with fractional flow reserve (FFR) requires that coronary resistance is stable and minimised. This is usually achieved by administration of pharmacological agents such as adenosine, which adds to the cost of the procedure and cannot be administered to all patients. In this study we determine (1) if there is a time when resistance is naturally minimised at rest and (2) assess the diagnostic efficiency, compared to FFR, of a new pressure-derived adenosine-free index of stenosis severity over that time. Methods 157 stenoses were assessed. In part 1 (39 stenoses), intracoronary pressure and flow-velocity were measured distal to the stenosis; in part 2 (118 stenoses), intracoronary pressure alone was measured. Measurements were made at baseline and under pharmacological vasodilatation with adenosine.
Results Wave intensity analysis identified a wave-free period where intracoronary resistance at rest is similar in variability and magnitude (coefficient of variation: $0.08 \pm 0.06$ and $284 \pm 147 \mathrm{~mm} \mathrm{Hg.s} / \mathrm{m}$ ) to those during FFR (coefficient of variation: $0.08 \pm 0.06$ and $302 \pm 315 \mathrm{~mm}$ Hg.s/m, $\mathrm{p}=\mathrm{NS}$ for both). The resting distal to proximal pressure ratio during this period, the instantaneous wave-Free Ratio (iFR), correlated closely with FFR $(\mathrm{r}=0.9, \mathrm{p}<0.001)$ with excellent diagnostic efficiency (receiver operating characteristic area under curve of $93 \%$, at FFR $<0.8$ ), specificity, sensitivity, negative and positive predictive values of $91 \%, 85 \%, 85 \%$ and $91 \%$, respectively.

Conclusion Intra-coronary resistance is naturally constant and minimised during a diastolic wave-free period. The instantaneous wave-Free Ratio calculated over this period produces a drug-free index of stenosis severity comparable to FFR. Adoption of instantaneous wave-Free Ratio would enable the benefits of physiologically guided angioplasty to be applicable to a larger patient population.

\section{COMPARISON OF FRACTIONAL FLOW RESERVE MEASUREMENTS OBTAINED USING CENTRAL VS DISTAL PERIPHERAL INTRAVENOUS ADENOSINE INFUSION TO INDUCE HYPERAEMIA}

doi:10.1136/heartjnl-2012-301877b.20

A Sirker, ${ }^{*}$ G Paul, R Dworakowski, N Melikian, J Byrne. King's College Hospital, London, UK

Introduction Measurement of fractional flow reserve (FFR) permits physiological evaluation of coronary lesions. Maximal hyperaemia is required and adenosine is most often used for this. The gold standard method is continuous adenosine infusion via a large central (usually femoral) vein. Use of radial access for coronary angiography is now used in over $50 \%$ of cases performed in the UK. Hence it is desirable to have an alternative route for adenosine delivery. Peripheral venous access is frequently obtained in the hand, since veins are often most readily accessible here. However concerns exist as to whether delivery from this site would achieve adequate vasodilatation. Our aim was to address this question.

Methods Ethical approval and informed consent was obtained. Subjects were selected from patients attending for coronary angiography who were deemed to need a pressure wire to assess an intermediate coronary stenosis. Subjects received intravenous adenosine infusion sequentially by two routes: first, via a 20G hand cannula, and then, after a washout period, via a $6 \mathrm{~F}$ femoral venous sheath. Adenosine was administered at $140 \mu \mathrm{g} / \mathrm{kg} / \mathrm{min}$ for each site. Data interpretation was performed in a blinded manner. Baseline values of FFR were recorded, as was the minimal FFR achieved with adenosine infusion, from each infusion site. Time to peak hyperaemia was also recorded separately for each infusion site.

Results 37 coronary artery lesions were evaluated in 23 patients. For the overall group, FFR using hand vein adenosine infusion was 0 . 86 \pm 0.09 ; FFR using femoral vein adenosine infusion was $0.85 \pm 0.09$. Individual paired comparisons of FFR readings using the different routes of adenosine administration are shown in Abstract 020 
figures 1 and 2 below. Abstract 020 figure 1 shows FFR using hand vein for adenosine vs FFR using femoral (groin) vein for adenosine-paired data are shown as ( $x, y)$ points. The line of unity is shown for a theoretical perfect agreement between the measures. Abstract 020 figure 2 shows a Bland Altman plot of FFR derived from hand vs groin adenosine infusions.

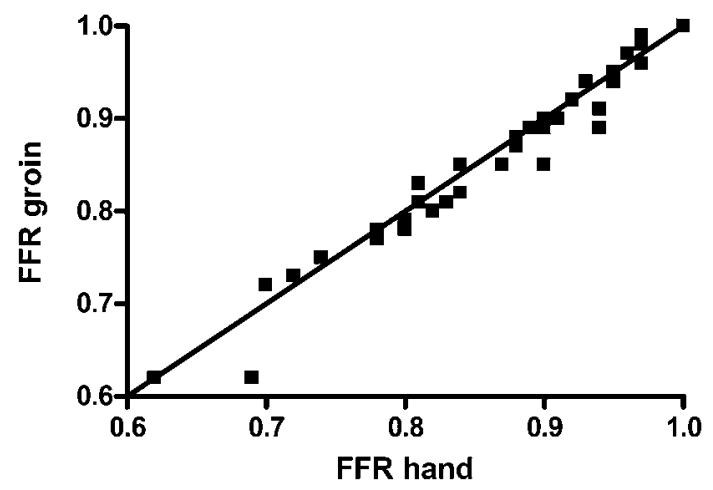

Abstract 020 Figure 1

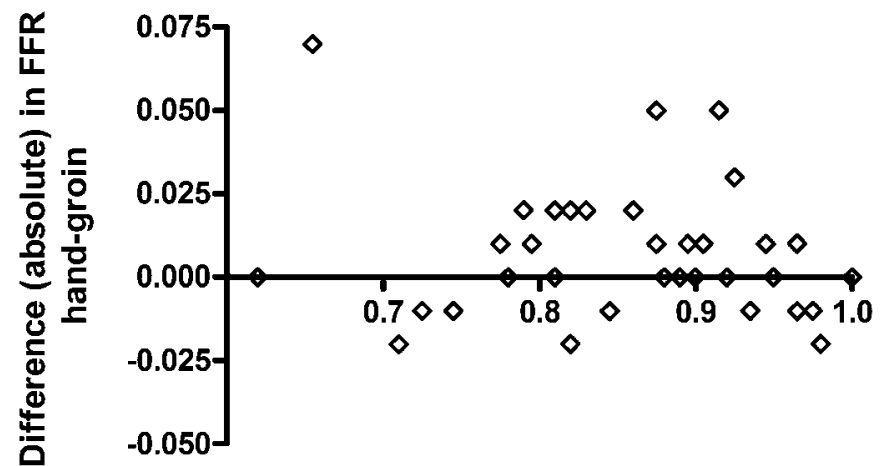

Average FFR from the two methods

Abstract 020 Figure 2

The mean difference between the two measures of FFR was 0.007 with a SD of 0.020 . The $95 \%$ limit of agreement extended from -0.031 to +0.046 . Using a threshold for ischaemia of FFR $\leq 0.75$, there were no cases in which use of hand vein adenosine would have misclassified a lesion compared to the "gold standard" central vein adenosine. Using a threshold for ischaemia of FFR $\leq 0.80$, there was one case which would have been classified differently. Time to maximal hyperaemia was significantly greater with hand vein adenosine infusion. ( $75.7 \pm 32.8 \mathrm{~s}$ vs $40.5 \pm 10.3 \mathrm{~s}, \mathrm{p}<0.001$ on $\mathrm{t}$ test). Conclusion The use of hand vein adenosine infusion produced very similar values of minimum FFR to those using femoral vein adenosine. These data have important practical implications for patients undergoing transradial procedures who require FFR assessment.

\section{COMPUTER MODELLED CORONARY PHYSIOLOGY AND "VIRTUAL" FRACTIONAL FLOW RESERVE FROM ROTATIONAL ANGIOGRAPHY}

doi:10.1136/heartjnl-2012-301877b.21

\footnotetext{
${ }^{1,2} \mathrm{P}$ D Morris, ${ }^{*}$ D R Ryan, ${ }^{1} \mathrm{R}$ Lycett, ${ }^{1,2} \mathrm{~A}$ C Morton, ${ }^{1} \mathrm{P}$ V Lawford, 'D R Hose, 1,2J Gunn. 'Department Cardiovascular Science, University of Sheffield, UK; ${ }^{2}$ Department of Cardiology, Northern General Hospital, UK
}

Background Percutaneous coronary intervention (PCI) guided by fractional flow reserve (FFR) measurement is superior to visual angiographic assessment alone. We have developed a workflow that takes a single rotational angiogram (RoCA), reconstructs the 3 dimensional arterial tree and applies computational fluid dynamics (CFD) to calculate the FFR without the need to induce hyperaemia or perform invasive pressure measurements.

Methods 20 patients, scheduled for elective PCI underwent RoCA. The FFR was measured with a Combowire (Volcano), under resting and hyperaemic conditions. Physiologically significant lesions were stented and the measurements repeated. The arterial anatomy was reconstructed on a Philips 3DCA workstation. Generic boundary conditions for CFD were derived from the measured data. The calculated ("virtual") and measured FFR values were then compared. Results There were 11 right coronary artery (RCA) cases ( 6 stented) and 12 left coronary artery (LCA) cases (8 stented). The anatomy was reconstructed, and the FFR computed in each case (pre- and post-stenting). The CFD model accurately predicted which lesions were physiologically significant (FFR $<0.8)$ and which were not (FFR $>0.8)$ in all cases. The virtual FFR values deviated from the measured by $\pm 6 \%(\mathrm{SD}=6 \%$ ) for both RCA and LCA cases.

Conclusion We have developed a novel, user-friendly workflow, which has the potential to predict FFR without the need for invasive measurements or inducing hyperaemic conditions. Our model identified lesions requiring intervention in all cases. Further work will optimise and refine the model by better characterising the downstream generic boundary conditions. We aim to improve the accuracy of the optimised model with more complex patients and lesions.

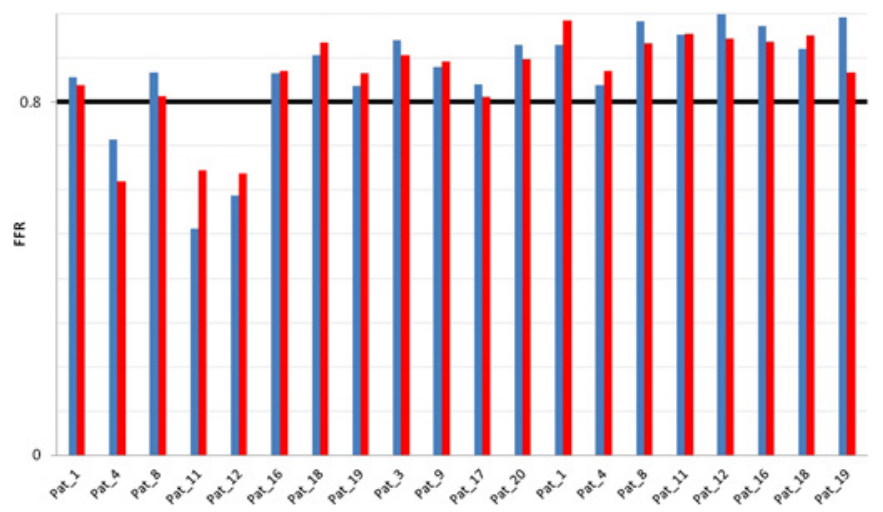

Abstract 021 Figure 1 Graph demonstrates measured FFR (blue) vs calculated FFR (red) for LCA cases (8 pre- and post-stent cases and 4 non-stented cases).

\section{DYNAMIC THREE-DIMENSIONAL WHOLE HEART MAGNETIC RESONANCE MYOCARDIAL PERFUSION IMAGING: VALIDATION AGAINST PRESSURE WIRE DERIVED FRACTIONAL FLOW RESERVE FOR THE DETECTION OF FLOW-LIMITING CORONARY HEART DISEASE}

doi:10.1136/heartjnl-2012-301877b.22

R Jogiva, ${ }^{*}$ G Morton, K De Silva, D Perera, S Redwood, S Kozerke, E Nagel, S Plein. Kings College London, London, UK

Background Three-dimensional (3D) myocardial perfusion cardiovascular magnetic resonance (CMR) has recently been proposed to overcome the limited spatial coverage of conventional perfusion CMR methods. The method has shown good diagnostic accuracy for the detection of coronary artery disease determined by quantitative coronary angiography. However the relationship between the severity of a coronary stenosis on quantitative coronary angiography and its functional significance is variable. Pressure wirederived fractional flow reserve (FFR) $<0.75$ correlates closely with objective evidence of reversible ischaemia and it has been demonstrated that ischaemia-guided PCI confers a prognostic benefit. 